\title{
EFFICIENCY OF USING PLATELET RICH FIBRIN PLUG FOR CLOSURE OF OROANTRAL FISTULA
}

\author{
Mohamed M. Shokry*
}

\begin{abstract}
Background: Oroantral fistula (OAF) may occur during dental extraction of maxillary teeth, surgical operations in the maxilla or due to massive maxillary tumors or cyst. Many techniques and materials have been suggested in the literature in order to close the OAF.
\end{abstract}

Aim of the study: to evaluate the efficiency of using the Platelet Rich Fibrin (PRF) as a plug for closure of oroantral fistula.

Methods: This clinical trial was conducted on twenty patients. Patients included within this study were of both gender, their age range from 29 to 45 years, suffered from established OAF. After de-coring of the fistula and curettage of the sinus lining, a Platelet Rich Fibrin (PRF) plug was prepared through collection and centrifuge of a ten $\mathrm{ml}$ of whole venous blood. The PRF plug was then secured to cover the OAF and sutured in place.

Results: Clinical results showed that the 20 cases had a favorable healing course with no signs of infection, no adverse tissue reaction following the operation with complete closure of the OAF. On CBCT, By measuring the fluid level at the site of oroantral fistula, the mean value of the fluid level preoperatively was $9.397 \mathrm{~mm} \pm 4.06$ then it decreased after six months with the mean value $2.48 \mathrm{~mm} \pm 0.96$. When comparing both pre and post-operative values of fluid level they were statically significant.

Conclusion: The use of PRF membrane biological plug is effective in closure of medium sized oroantral fistulae. This method had proven usefulness, safety, availability in an easy-to-develop manner and finally its low cost.

KEYWORDS: Oroantral fistula, Platelet rich fibrin, Maxillary sinus.

\section{INTRODUCTION}

Maxillary sinus surgeries are frequently operated by the oral and maxillofacial surgeons. The maxillary sinus enlarges from a small cavity during the third month of intra-uterine life, reaching its full development nearly by the age of eighteen years old. Its volume ranges from $20-25 \mathrm{ml}$ in adults. The alveolar process and the hard palate form the floor of the maxillary sinus. ${ }^{(1)}$

\footnotetext{
* Lecturer of Oral and Maxillofacial Surgery, Faculty of Dentistry, Alexandria University, Egypt.
} 
Maxillary premolars and molars roots are in close vicinity to the floor of the sinus and may be present within it in some patients. In literature, the most common etiological factor for oroantral fistula is the traumatic extractions of posterior molars especially the first molars, followed by extraction of the upper premolars and the second molars. ${ }^{(2-4)}$

Therefore, oroantral communication (OAC) and subsequent development of an oroantral fistula (OAF) is a common post-extraction complication of the upper posterior teeth or surgical operations within the maxilla. If the patient has a healthy sinus, an OAC less than 4 to $5 \mathrm{~mm}$ in diameter will most likely heal spontaneously. ${ }^{(5)}$

If the communication is not detected and managed properly, the risk of development of an epithelialized permanent oroantral fistula (OAF) is high. The resultant fistula keeps the sinus open; it leads to the passage of bacteria from the oral cavity into the maxillary sinus and the development of inflammation, maxillary sinusitis, and infection with all possible consequences. In few cases, closure of the fistula can occur spontaneously by swelling of the overlying gingiva. ${ }^{(5,6)}$

OAF is a pathological communication between the epithelium of the oral cavity and that of the maxillary sinus, which may has different origins as from iatrogenic complications, dental infections, radiotherapy, osteomyelitis, and trauma or after implant insertion. When OAF takes place together with the presence of larger perforations, surgery becomes the only choice of treatment for prevention of chronic and irreversible changes in the maxillary sinus ${ }^{(5-9)}$.

The absence of any pathology within the sinus and the selection of the appropriate surgical technique will lead to successful closure of the OAF. Several methods have been proposed for its closure including buccal, palatal flaps and their modifications. ${ }^{(7)}$ The preferred line of treatment varies between surgeons, upon their previous experience. In addition to the commonly known techniques for the closure of OAF, some alloplastic materials have also been introduced. Moreover, with the progressing technology allo-transplants as fascia lata and dura mater, have also been used for closure of OAF. ${ }^{(8,9)}$

The closure of large defects occurred due to a massive trauma or tumors by the aid of local flaps is a challenging procedure and may lead to failure. Flaps from forehead, extremities or tongue have been described earlier in the literature ${ }^{(5-9)}$. The buccal pad of fat has become popular for the closure of large oroantral defects. ${ }^{(10)}$

Lately, Platelet Rich Fibrin (PRF) has been widely applied in various fields of dentistry. It consists of a fibrin matrix that includes platelet cytokines and growth factors. These components may be freed and can serve as a resorbable membrane. ${ }^{(1)}$ Choukroun and his co-workers ${ }^{(11-13)}$ were the pioneers for applying PRF protocol in oral and maxillofacial surgery to enhance bone healing in implantology. PRF is considered and well-thought nowadays to be a healing biomaterial. ${ }^{(12)}$ It is likely that the inflammatory regulation noted on surgical sites treated with PRF is the result of the effects of cytokines trapped within the fibrin network and released during the initial remodeling phase. ${ }^{(13)}$

In this study, an innovative idea was evaluated through the use of PRF as a plug closing a long standing oroantral fistula. This work presents an attempt to assess both clinically and radiographically the closure of an OAF using PRF plug.

\section{PATIENTS AND METHODS}

This study was carried out as a clinical trial. The sample size was calculated using http://epitools. ausvet.com.au by taking the means and variance of OAF size from a previous study conducted by Visscher et al ${ }^{(8)}$. Assuming a confidence level of $95 \%$ and a study power of $80 \%$, the estimated sample size was calculated to be 16 patients. In order to 
avoid the drop out from the sample, twenty patients suffering from long standing oroantral fistula in maxillary premolars or molars region were selected from those attending the out-patient clinics of Oral and Maxillofacial Surgery Department, Faculty of Dentistry, Alexandria University. All patients were treated in the Oral and Maxillofacial Surgery Department, Faculty of Dentistry, Alexandria University. This study was approved by the ethics committee, and an informed consent was obtained from all patients before their inclusion in the study.

The diagnosis and hence the patients selection were based on both clinical and radiographic examinations. Patients included within this study were of both gender ( 8 females and 12 males), their age range from 29 to 45 years, suffered from established OAF $>5 \mathrm{~mm}$ in diameter and the condition of the sinus needed to be operated by Caldwell-Luc procedure. They were selected to be free from any systemic or blood disorders that may interfere with the preparation of PRF. Smokers, pregnant, lactating females and medically debilitated patients were excluded from this study.

\section{Pre-operative phase}

A detailed medical history was recorded for all patients, as well as the cause and time elapsed since the discovery of the OAF. Also, the history of any previous attempts to close the OAF was noted. A cone beam computerized tomography scan (CBCT) (KODAK CS 3D Imaging) was also performed preoperatively to confirm the clinical situation, to examine the condition of the maxillary sinus and to measure the intra-sinus fluid level serving as a baseline measurement and finally to measure the exact size of the fistula. (Figure. 1)

Furthermore, irrigation with warm saline solution through the fistula path for 1 week (three times daily) was performed in an attempt to decrease the amount of infection within the sinus. Prophylactic procedures including taking $2 \mathrm{~g}$ amoxicillin

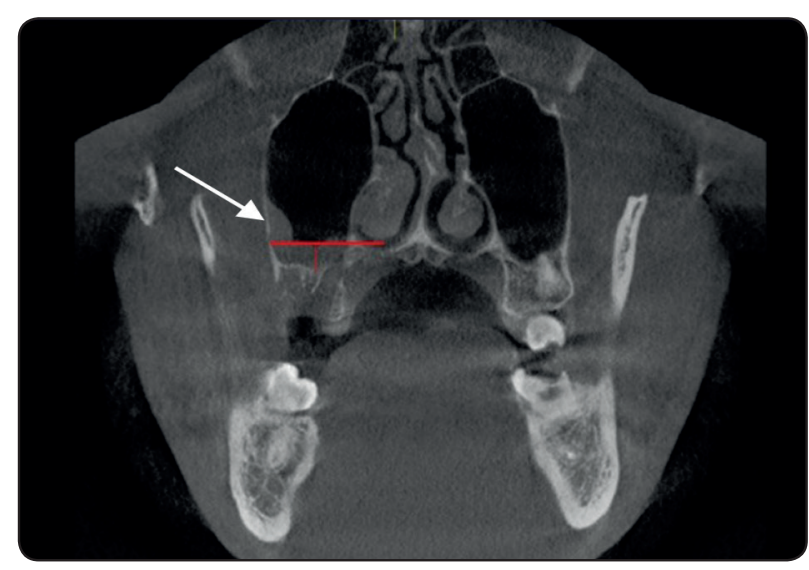

Fig. (1) preoperative CBCT showing the fluid level in the site of the OAF (arrow)

antibiotic (Augmentin by GlaxoSmithKline (GSK) one hour before surgery and mouth rinsing with $0.12 \%$ chlorhexidine gluconate solution $(\mathrm{G} \bullet \mathrm{U} \bullet \mathrm{M} \circledast$ PAROEX ${ }^{\mathrm{TM}}$ ) were prescribed.

\section{Operative phase}

All operations were carried under general anesthesia and under complete aseptic conditions. A circular incision with $3 \mathrm{~mm}$ margin was made around the OAF by the aid of Bard-Parker blade number 15. Then the epithelial tract and any inflammatory tissue within the opening of the OAF were completely removed and excised (de-coring). This step was followed by the reflection of a full thickness trapezoidal mucoperiosteal flap extending from the incisor region to the molar area in the side of the fistula. Access to the maxillary sinus is gained through the fistula in four cases, whereas in sixteen cases the maxillary sinus was entered through a Caldwell-Luc opening just lateral to the canine fossa. Curettage, removal of the maxillary sinus lining, debridement and removal of any foreign material within the sinus was done. Irrigation of the sinus with normal saline is finally performed. Intranasal antrostomy was not required in any of the cases. (Figure 2a, b) 


\section{Protocol for PRF preparation}

PRF was prepared according to choukroun (12) from the patient's own blood; almost $10 \mathrm{ml}$ of whole venous blood; $5 \mathrm{ml}$ in each of the two sterile test tubes $(6 \mathrm{ml})$ without anticoagulant was collected. Immediate centrifugation was done at 3,000 revolutions per minute (RPM) for 10 minutes. At the end of centrifugation process, the tubes were removed, sterile tweezers were inserted into the tubes to grab the fibrin clots with attached RBCs which were scrapped away by a smooth spatula and discarded. PRF membrane was then obtained by driving out the serum from the clot which was applied to cover the OAF. The flap was then closed after performing a horizontal incision in the base of the flap in order to facilitate the advancement of the flap and to cover the OAF and the PRF plug which was stabilized to the flap by a suture. Suturing of the flap was finally done using 4/0 vicryl mattress suture. (Figure 3 a, b)

\section{Post-operative and follow-up phase}

All patients were given oral amoxicillin with clavulanic acid $1000 \mathrm{mg}$ (Augmentin by GlaxoSmithKline, UK) twice daily for five days, Ibuprofen $400 \mathrm{mg}$ (Bruffen by Abbott USA), three times daily after meals for four days and decongestant nasal drops and were advised to apply ice packs extraorally at the surgical side the day of surgery, to stop smoking, to avoid strong sneezing, use a pipette during drinking, and to eat soft diet for one week. All patients were followed up every

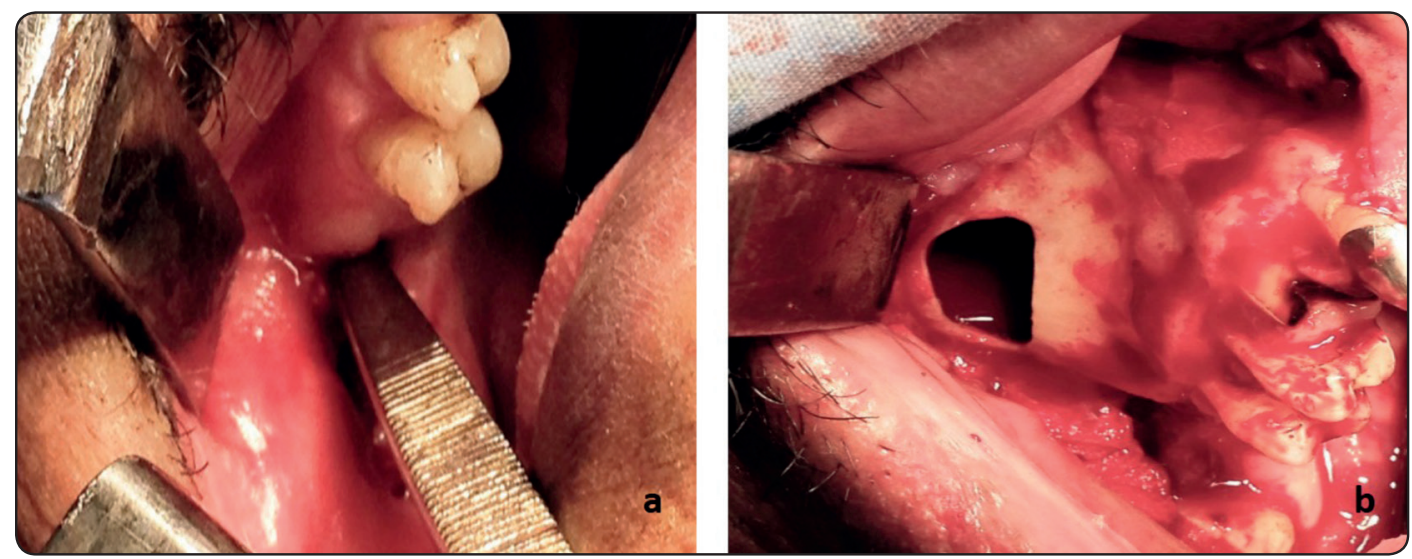

Fig. (2) a) The long standing OAF in the maxillary first molar region. b) a full thickness flap reflection and a Caldwell-Luc opening at the site of the OAF

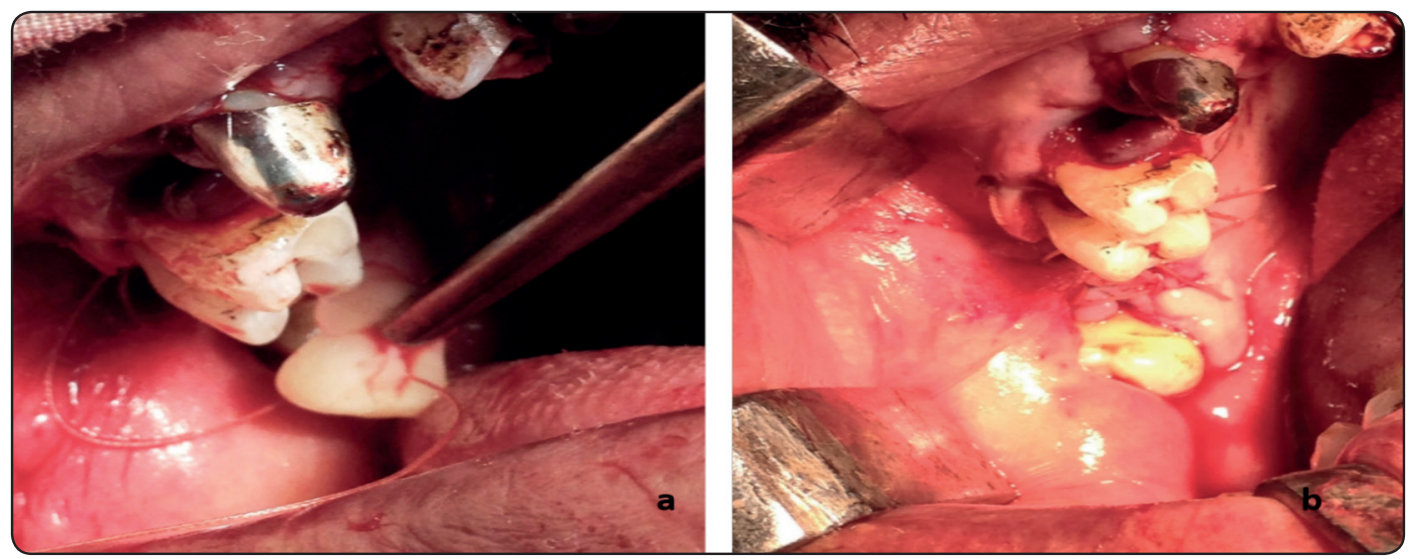

Fig. (3) a) PRF membrane plug cover the OAF. b) Suturing of the flap over the PRF plug and the OAF 
week during the first post-surgical month and then re-checked monthly for three months. The clinical variables evaluated during the follow up periods consist of postoperative pain, swelling, infection and wound healing. On the other hand, radiographically a CBCT was requested at the sixth months follow up interval to examine the condition of the sinus and the intra-sinus fluid level and to ensure the complete closure of the OAF.

All the gathered data were summarized and tabulated and represented in suitable graphs. Statistical analysis were performed by the aid of Social Package of Social Science software (SPSS version 21.0)

\section{RESULTS}

This study involved 20 patients suffering from long standing OAF with diameter $>5 \mathrm{~mm}$. The etiology of the OAF was traumatic tooth extractions; 15 patients had the OAF in the maxillary first molar region, 3 patients in the maxillary second molar region and 2 patients suffered from OAF in the second premolar area. (Figure 4)

Eight (8) patients included within this study were female patients $(40 \%)$, while the other twelve (12) patients were male patients $(60 \%)$. Their age ranged between 29-46 years with mean \pm SD of $35.13 \pm 7.97$ years.

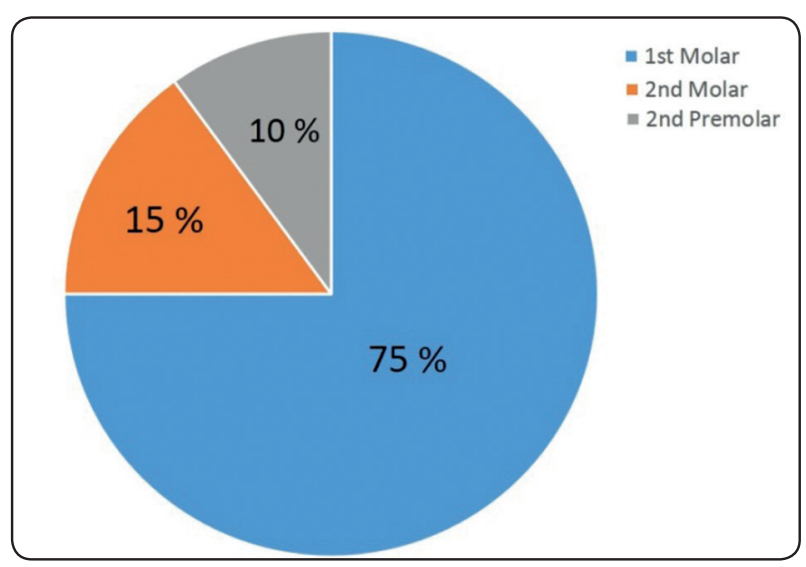

Fig. (4) Pie chart graph showing sample distribution according to the etiological tooth for the OAF
Clinical examination of patients starts at the first post-operative week; results showed that the 20 cases had a favorable healing course with no signs of infection, no adverse tissue reaction following the operation with complete closure of the $\mathrm{OAF}$ and normal color of the overlying mucosa was detected throughout the follow-up period which was extended till the $3^{\text {rd }}$ post-operative month. There was complete closure of the OAF in all cases and no recurrence has been detected. The color and texture of the flap compared with the surrounding tissue were normal. No breaking down at the operation site or inward collapse of the tissues or any abnormal tissue reaction during the follow up period was encountered. Furthermore, the wounds became successfully epithelialized in 2 to 3 weeks post-operatively. No bleeding and proper hemostasis was observed at the time of discharge. (Figure 5)

Regarding the developed post-operative complications, one of the patients (5\%) complained from numbness in the region supplied by the infraorbital nerve in the next day of the operation that resolved gradually within 6 weeks postoperatively. Additionally, in only two cases (10\%) vestibular sulcus height was reduced permanently.

Radiographically, The CBCT taken preoperatively revealed the presence of defect in the floor of the maxillary sinus, the size of oroantral fistula ranged between 5.1-8.7mm (mean 6.43 \pm 1.03 ).

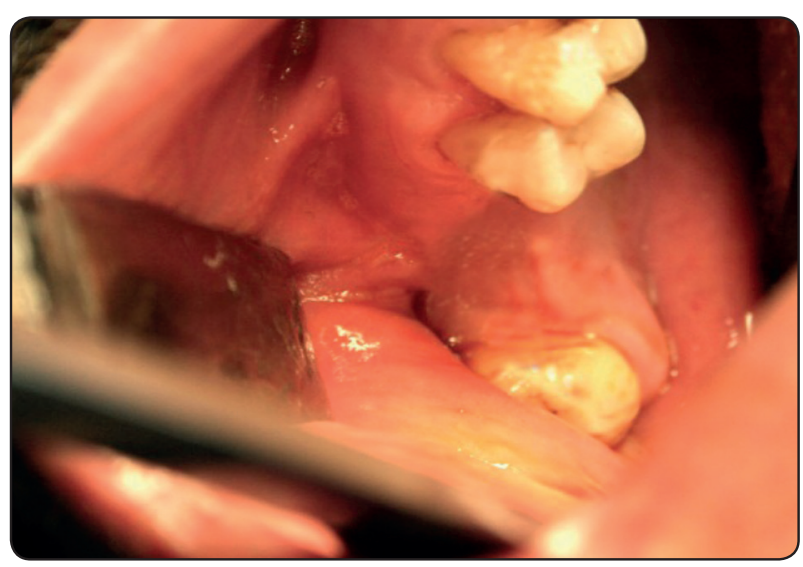

Fig. (5) Two weeks Post-operative acceptable healing and closure of the OAF 
Furthermore, CBCT showed that 14 patients suffered from polypoids in the affected maxillary sinus and only 6 cases had remaining root escaped inside the maxillary sinus.

By measuring the fluid level within the maxillary sinus at the site of oroantral fistula, its mean value preoperatively was $9.397 \mathrm{~mm} \pm 4.06$ then it decreased six months following surgery to be 2.48 $\mathrm{mm} \pm 0.96$. When comparing both values, they were statically significant. (Figure 6)

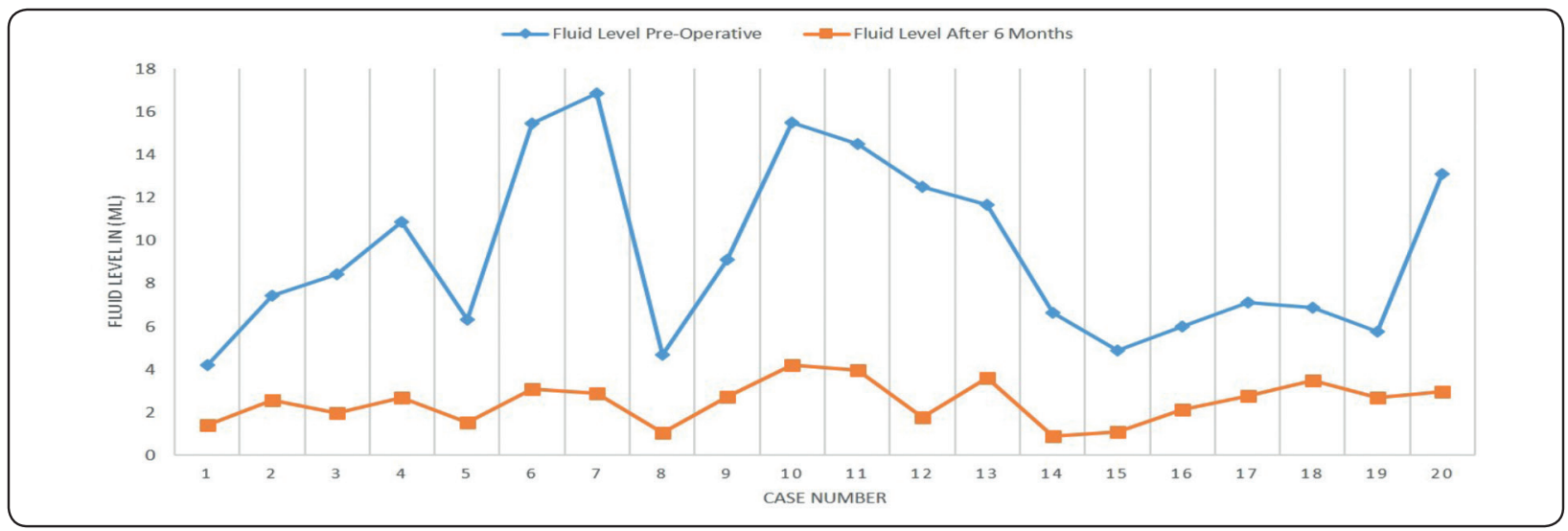

Fig. (6) Line chart graph showing the decrease in fluid level within the sinus at $6^{\text {th }}$ months postoperatively

\section{DISCUSSION}

Oroantral communication with consequent establishment of an OAF is a clinical complication commonly encountered by oral and maxillofacial surgeons. ${ }^{(6)}$

Yilmaz et al ${ }^{(14)}$, mentioned that fistulas are common in male than in female, this may be attributed to more traumatic tooth extraction in male, which also agrees with our findings. The obtained results run parallel to studies accomplished by Von Wowern ${ }^{(15)}$, Killey and Kay ${ }^{(16)}$, Punwutikorn et al. (17) and Hanazawa et al ${ }^{(18)}$, who stated that the most frequent cause of the OAF was tooth extraction. In this study, the highest incidence of OAF was found following extraction of the first molar followed by the second premolar.

The findings of this study regarding the age of OAF occurrence is matched and agreed with the reports of other investigations ${ }^{(8-10,14)}$. OAF usually happens after the third decade of life. Larger sinuses are seen in elderly patients with few maxillary teeth than younger individuals. In this study, the highest frequency was found in the 32-39 years age group. ${ }^{(18-20)}$

A small oroantral communication smaller than $2 \mathrm{~mm}$ in diameter resulting from tooth extraction will usually close spontaneously if the sinus is patent and the blood clot fills the socket. However, defects that are larger than $5 \mathrm{~mm}$ in diameter rarely heal spontaneously and usually will require surgical intervention for closure. ${ }^{(21)}$ Also, in a study conducted by Von Wowern ${ }^{(19)}$ on OAC closure, he assumed that there is a very little chance of its selfhealing, and thus surgical closure of a perforation is frequently required.

Various procedures have been proposed for the surgical repair of OAF. Buccal advancement and palatal pedicle flaps are the most commonly used. ${ }^{(7)}$

Closure of small OAF $(<5 \mathrm{~mm})$ and immediate OAC is usually done by the aid of buccal flaps with horizontal periosteal incisions. However, this may results in a shallow vestibular sulcus, which 
can affects prosthodontics rehabilitation and oral hygiene maintenance, this problem currently solved with the availability of implant-retained overdentures. ${ }^{(6,7)}$

On other hand, palatal flaps are used commonly to close larger size fistulae. They are based on the greater palatine artery, and its integrity has been considered an important success factor. ${ }^{(7)}$

Several materials have been used in order to close the OAF. Results obtained from using such materials run within the same line of the results reached from this work. Hanazawa et al ${ }^{(18)}$, and later Dolanmaz et al ${ }^{(10)}$ closed an oroantral communication through the use of a pedicled buccal pad of fat graft. This technique is useful in patients with a fistula of $8-20$ $\mathrm{mm}$ in diameter. After three weeks, the fatty tissue turns into granulation tissue and epithelizes, which has been proven by histological indicators. Similar clinical results have been reached from this study.

Furthermore, in 2012, Cankaya et al ${ }^{(22)}$, used an autogenous cartilage graft harvested from the ear to close the bone defect of a persistent OAF, and soft tissue closure was achieved with a palatal rotational flap.

Similar clinical and radiographic outcomes have been reached by Burić in who investigated the use of N-butyl cyanoacrylate with metacryloxisulfolane (Glubran 2) synthetic surgical glue for the nonsurgical closure of oroantral communication. Two months following closure of OACs, he stated that they were successfully closed when evaluated by panoramic and Water's view radiography. ${ }^{(23)}$

This study was done to evaluate the efficiency of using Platelet-rich fibrin (PRF) as a plug for closure of long standing OAF. It as an autologous fibrin matrix which can be classified as a second generation platelet concentrate as it is consisted of leukocytes and does not need an anticoagulant. The acceptable clinical and radiographic results obtained within this work may be attributed to the slow release of growth factors such as transforming growth factor $\beta 1$, platelet-derived growth factor $\beta$, and vascular endothelial growth factor, especially during the first month from the PRF plug as demonstrated in previous study conducted by Choukroun et al. ${ }^{(12)}$

When using this autologous material technique for closure of OAF, it can be said that no vestibular sulcus narrowing that may be seen in buccal advancement flap cases occurs- Moreover, while this technique allows healing of oral mucosa, it also protects its normal anatomic architecture. Thus, the need for future operations could be eliminated in patients who may use a removable prosthesis. Unlike palatal flap cases, there are no secondary healing areas that require a long and painful healing period. Additionally, it may be suggested that this is an easier method when the operator has equal experience and skills with this technique as the other techniques.

\section{CONCLUSIONS}

It can be concluded that PRF membrane biological plug is effective in closure of medium sized oroantral fistulae. The most remarkable advantages of this procedure are, the simplicity and quick surgical technique, few complications and its highly predictable results without any esthetic sequel. The PRF membrane is biologically competent and it is easy to use. This method had proven usefulness, safety, availability in an easy-to-develop manner and finally its low cost. Also it showed excellent hemostasis, predictable flap adaptation and enhanced wound healing. Finally, management of OAF using PRF membrane biological plug is an acceptable and reliable alternative and can be used as a primary treatment choice by experienced surgeons.

\section{REFERENCES}

1. Gonty AA. Diagnosis and management of sinus disease. In Principles of oral and maxillofacial surgery (Vol. 1, pp. 249-266). Lippincott Philadelphia, PA, 1992.

2. Kilic C, Kamburoglu K, Yuksel SP, Ozen T. An Assessment of the Relationship between the Maxillary Sinus Floor and the Maxillary Posterior Teeth Root Tips Using Dental Cone-beam Computerized Tomography. Eur J Dent.; 4(4):462-67, 2010. 
3. Von Arx T, Fodich I, Bornstein MM. Proximity of premolar roots to maxillary sinus: a radiographic survey using cone-beam computed tomography. J Endod.; 40(10): $1541-48,2014$.

4. Shokri A, Lari S, Yousef F, Hashemi L. Assessment of the relationship between the maxillary sinus floor and maxillary posterior teeth roots using cone beam computed tomography. J Contemp Dent Pract.; 15(5):618-22,2014.

5. Abuabara A, Cortez AL, Passeri LA, de Moraes M, Moreira RW. Evaluation of different treatments for oroantral/oronasal communications: experience of 112 cases. Int J Oral Maxillofac Surg.; 35(2):155-58,2006.

6. Güven O. A clinical study on oroantral fistulae. J Craniomaxillofac Surg.; 26(4):267-71,1998.

7. Borgonovo AE, Berardinelli FV, Favale M, Maiorana C. Surgical options in oroantral fistula treatment. Open Dent J. ; 6:94-8,2012.

8. Visscher SH, van Minnen B, Bos RR. Closure of oroantral communications: a review of the literature. J Oral Maxillofac Surg. ; 68:1384-91,2010.

9. Awang MN. Closure of oroantral fistula. Int J Oral Maxillofac Surg. ; 17(2):110-5,1988.

10. Dolanmaz D, Tuz H, Bayraktar S, Metin M, Erdem E, Baykul T. Use of pedicled buccal fat pad in the closure of oroantral communication: Analysis of 75 cases. Quintessence Int; 35:241-46,2004.

11. Saluja H, Dehane V, Mahindra U. Platelet-Rich fibrin: A second generation platelet concentrate and a new friend of oral and maxillofacial surgeons. Ann Maxillofac Surg.; 1(1): 53-7, 2011.

12. Choukroun J, Adda F, Schoeffler C, Vervelle A. Une opportunité en paro-implantologie: Le PRF. Implantodontie; 42:55-62, 2001.
13. Naik B, Karunakar P, Jayadev M, Marshal R. Role of Platelet rich fibrin in wound healing: A critical review. J Conserv Dent.; 16(4): 284-93,2013.

14. Yilmaz T, Suslu AE, Gursel B. Treatment of oroantral fistula: experience with 27 cases. Am J Otolaryngol; 24: 221- 3, 2003.

15. Von Wowern N. Correlation between the development of an oroantral fistula and the size of the corresponding bony defect. J Oral Surg; 31:98-102,1973.

16. Killy HC, Key LW. The maxillary sinus and its dental implications. John Wright \& Sons Ltd, Bristol London; $1-2,1975$

17. Punwutikorn J, Waikakul A, Pairuchvej V. Clinically significant oroantral communications- a study of incidence and site. Int J Oral Maxillofac Surg; 23:19-21,1994.

18. Hanazawa Y, Itoh K, Mabashi T. Closure of oroantral communications using a pedicled buccal fat pad graft. J Oral Maxillofac Surg; 53: 771-75,1995.

19. Von Wowen N. Oroantral communications and displacement of roots into the maxillary sinus: a follow-up of 231 cases. J Oral Surg; 29:622-27,1971.

20. Ehrl PA. Oroantral communication: epicritical study of 175 patients, with special concern to secondary operative closure. Int J Oral Surg; 9:351-58,1980.

21. Skoglund LA, Pedersen SS, Hoist E. Surgical management of 85 perforations to the maxillary sinus. Int. J. Oral Surg; 12:1-5,1983.

22. Cankaya A, Erdem M, Cakarer S, Isler S, Demircan S, Korhan C. Reliability of Two Surgical Methods for Oroantral Communication Closure; A Clinical Study of 20 Patients. Otolaryngology; 2:113, 2012.

23. Burić N. Use of N-butyl cyanoacrylate with metacryloxisulfolane (glubran 2) surgical glue for flapless closure of oroantral communication. Implant Dent.; 22(3):238-43, 2013. 